\title{
Freezing of Lennard-Jones fluid in cylindrical nanopores under tensile conditions
}

\author{
Hideki Kanda · Minoru Miyahara
}

Received: 15 June 2007 / Revised: 25 September 2007 / Accepted: 25 September 2007 / Published online: 16 October 2007

(C) Springer Science+Business Media, LLC 2007

\begin{abstract}
We have shown the Lennard-Jones (LJ) phase diagram for a slit-shaped nanopore by molecular simulations and thermodynamically predicted the results with no adjustable parameter. With this success, LJ phase diagrams are predictable. In this study, the freezing of an $\mathrm{LJ} \mathrm{CH}_{4}$ capillary condensate under a tensile condition in a nonstructural carbon nanopore with a cylindrical geometry was examined using molecular dynamics (MD) simulation. We employ a unit cell in contact with a bulk vapor phase, which is useful for the determination of the bulk vapor pressure in equilibrium with the molecules in a pore. The MD simulation results show liquid-solid (amorphous) phase transitions with a variation in the bulk vapor pressure. The frozen particles are arranged in concentric circular regions along the wall similar to those reported by Maddox and Gubbins. The freezing points are determined from the variations in density, enthalpy, arrangement, and structural functions. The obtained liquid-solid coexistence points are found to exhibit a significant dependence of the freezing point on the equilibrium bulk vapor pressure, forming an extraordinarily skewed curve on the p-T diagram, in contrast to the bulk phase coexistence that is represented by an almost vertical line. The origin of the significant dependence is considered to be the Laplace effect on the capillary condensate similar to the case with a slit-shaped pore. A simple model, which the authors
\end{abstract}

H. Kanda $(\bowtie)$

Energy Engineering Research Laboratory, Central Research Institute of Electric Power Industry, Yokosuka,

Kanagawa 240-0196, Japan

e-mail: kanda@criepi.denken.or.jp

M. Miyahara

Chemical Engineering Department, Kyoto University,

Kyoto 615-8510, Japan earlier presented for slit-shaped nanopores, successfully predicted the $\mathrm{p}-\mathrm{T}$ relation of the freezing point for cylindrical nanopores as well.

Keywords Solidification - Thermodynamic model . Molecular dynamics simulation

\section{Introduction}

The freezing points of the fluid confined in porous solids are usually lowered (Gelb et al. 1999). However, recently, it was clarified that the solidification of pore fluid is more complex than the common knowledge and the breakdown of micropores according to the Gibbs-Thomson equation. Klein and Kumacheva (Klein and Kumacheva 1995, 1998; Kumacheva and Klein 1998) observed the freezing point elevation of the cyclohexane and octamethylcyclotetrasiloxane (OMCTS) confined between parallel mica plates in a surface force apparatus. Moreover, many experimental studies (Kaneko et al. 1999; Watanabe et al. 1999; SliwinskaBartkowiak et al. 2001a, 2001b; Miyahara et al. 2002) have also reported the freezing point elevation. In addition, Miyahara and Gubbins (Miyahara and Gubbins 1997) theoretically conducted a grand canonical Monte Carlo (GCMC) simulation of solid-liquid phase transition of Lennard-Jones (LJ) methane between parallel graphite s plates. In general, the freezing points depend on the strength of the attractive potential energy from pore walls, the fluid in a slit pore shows a freezing point elevation as well as depression; the critical strength to divide these two cases is the potential energy exerted by the solid state of the fluid. Maddox and Gubbins (Maddox and Gubbins 1997) and the authors (Kanda et al. 2000) studied the solidification of LJ methane within carbon cylindrical nanopores using molecular dynamics (MD) 
and GCMC simulations. They found a nonmonotonic dependence of the pore diameter on a geometric constraint of the cylindrical wall. The geometrical constraint, which originated from a solid-like (amorphous) phase in the cylindrical pore relative to the fcc lattice phase, is confirmed to become stronger for a smaller pore size. The latent heat is also smaller for smaller pore sizes. In addition, the authors (Miyahara et al. 2000; Kanda et al. 2004) investigated the freezing of capillary condensed LJ methane below the saturated pressure by the MD simulation. The obtained freezing points significantly depended on the equilibrium bulk-phase pressure, forming an extraordinarily skewed curve on a $\mathrm{p}-\mathrm{T}$ diagram, in contrast to the bulk-phase coexistence shown by an almost vertical line (Clapeyron equation). This was interpreted as a result of Laplace effect. The inner pressure of the capillary condensate is lowered to a negative value due to the strong Laplace effect. The authors presented a simple model in which the Clapeyron equation was applied to the inner pressure. It predicted the freezing point depression of the capillary condensate. We have shown the Lennard-Jones (LJ) phase diagram for a slit-shaped nanopore by molecular simulations and thermodynamically predicted the results with no adjustable parameter. With this success, complete LJ phase diagrams are predictable (Kanda et al. 2005; Kanda and Miyahara 2007).

In this study, we investigate the freezing of the capillary condensed LJ methane in carbon cylindrical nanopores below the saturation pressure by the MD simulations. The reliability of the authors' simple model for slit-shaped pores is tested with the simulation results under various temperatures or pressures. Note that the purpose of this study is not to compare with a real system, but with a thermodynamic model.

\section{MD simulation with imaginary bulk vapor phase}

We employ the NVT-MD technique with an imaginary vapor phase to obtain the variations of the bulk vapor pressure. Since this technique is described in detail in Refs. (Miyahara et al. 2000; Kanda et al. 2004, 2005), the features of the simulation are briefly explained below. Additional information on this topic can be obtained from the Refs. (Miyahara et al. 2000; Kanda et al. 2004, 2005). As shown in Fig. 1, the middle of the cell is the pore space with a given potential energy (full potential field: FPF). At each end of the cell, at a distance from the edge of the FPF, we set a border with an imaginary bulk vapor phase. Since the potential energy of the pore wall must be zero in the bulk vapor phase, there should exist a connecting space with the slope of the potential energy between the bulk vapor phase and the pore space; this space is termed as the potential buffering field (PBF). The benefit of this simulation cell is reflected in the easy

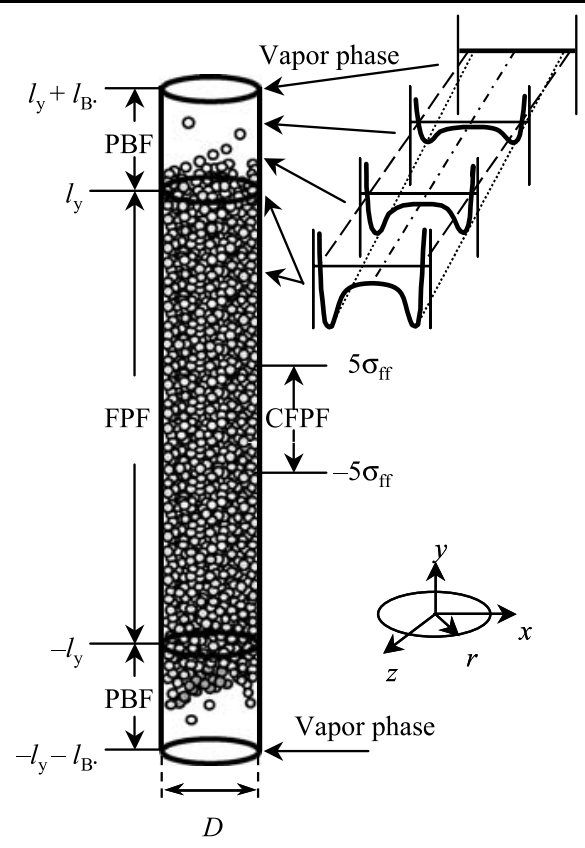

Fig. 1 Unit cell and potential profile within PBF

determination of the equilibrium vapor pressure. The molecules attempting to desorb from the pore space must climb the potential slope in the PBF, and only those with sufficient kinetic energy can reach the border plane. If we set a perfect reflection condition at the border, the frequency of the particles moving upward should be a direct measure of the vapor pressure in bulk that is in equilibrium with a given adsorbed phase. Note here that the FPF remains filled with a condensed phase as far as the equilibrium pressure is larger than the condensation pressure.

Methane was modeled using a truncated and shifted LJ $(12,6)$ potential: $\varepsilon_{\mathrm{ff}} / k=148.1 \mathrm{~K}$ and $\sigma_{\mathrm{ff}}=0.381 \mathrm{~nm}$. The cut-off distance of the adsorbate was set to be $5 \sigma_{\mathrm{ff}}$, which was considered to be large enough to represent the particles with a full $\mathrm{LJ}$ potential. Thus, no long-range correction was attempted. For the pore potential, we used that for the cylindrical pore surrounded by a semi-infinite solid, which was derived by Peterson et al. (Peterson et al. 1986) with a cylindrical coordinate integration. At a radial position $r$, the interaction between a fluid particle and the pore wall with radius $\mathrm{R}$ is given as follows:

$\phi_{\mathrm{fs}}(r, R)=\pi \varepsilon_{\mathrm{fs}} \rho_{\mathrm{s}}\left[\frac{7 \sigma_{\mathrm{fs}}^{12}}{32} K_{9}(r, R)-\sigma_{\mathrm{fs}}^{6} K_{3}(r, R)\right]$

where

$$
\begin{aligned}
& K_{n}(r, R) \\
& \quad=R^{-n} \int_{0}^{\pi} d \Theta\left[-\frac{r}{R} \cos \Theta+\left(1-\left(\frac{r}{R}\right)^{2} \sin ^{2} \Theta\right)^{1 / 2}\right]^{-n}
\end{aligned}
$$


Here, $r$ is the distance between a fluid particle and the center of the wall surface atoms, and $\rho_{\mathrm{s}}$ is the number density of the interaction sites in the carbon wall being $1.14 \times 1029 \mathrm{~m}^{-3}$. The values of the parameters $\varepsilon_{\mathrm{ss}} / k$ and $\sigma_{\mathrm{ss}}$ were $28.0 \mathrm{~K}$ and $0.340 \mathrm{~nm}$, respectively, and the Lorentz-Berthelot combining rules were applied to obtain parameters for the methane-carbon interactions $\varepsilon_{\mathrm{fs}} / k$ and $\sigma_{\mathrm{fs}}$. The other constants are as follows: the mass of methane $m=2.665 \times 10^{-26} \mathrm{~kg}$. The pore diameter $\mathrm{D}$ ranges from $5.5 \sigma_{\mathrm{ff}}$ to $9.5 \sigma_{\mathrm{ff}}$.

In the PBF, the potential within the FPF was linearly attenuated towards zero at the border of the imaginary vapor phase. The pore fluid mostly remains in the FPF under a lower equilibrium pressure, but its edge enters the PBF when simulating the pore fluid with a higher equilibrium pressure. Maximum attention was paid to maintain a sufficient distance between the border plane and the edge of the pore fluid so that a particle in the vapor-phase plane does not receive any interactions from the condensate in the pore. If the PBF length $l_{B}$ is insufficient, resultant equilibrium pressure suffers from overestimation because less number of particles, in reality, should deserve to stand for "ideal-vapor particles free from fluid's potential," as examined previously.

The existence of an interface between the pore fluid and vapor phase in the cell, instead of the usually employed periodic boundary condition in the y-direction, brings another feature. For a condition in which the pore fluid should freeze, the central portion of the FPF (CFPF) holds the frozen phase; however, a melted phase exists around the edge of the condensate in the PBF because of the weaker potential field and because of somewhat parallel case with so called "surface melting." This melted phase may, to some extent, affect the structure of the solid phase in the outer portion of the FPF. We should set a sufficient length of the FPF to accommodate this intermediate phase in addition to the CFPF. With a shorter length of the FPF, the solid phase may not sustain even under a favorable condition. With a sufficient length of the FPF, a data analysis was made for the fluid particles in the CFPF, between $-5 \sigma_{\mathrm{ff}}<y<5 \sigma_{\mathrm{ff}}$, so as to avoid being affected by the melted phase near the interface. Considering the above influences, we determined the following settings. The length of the FPF, 2ly , was 7D and that of the PBF, $1_{\mathrm{B}}$, was $13.12 \sigma_{\mathrm{ff}}(5 \mathrm{~nm})$.

A simulation run for a given number of fluid particles $\mathrm{N}$ started from an initial configuration arranged in the form of a liquid-like phase. In the beginning, the temperature of the system was controlled by velocity scaling once every 100 steps for 1000 ps. The leapfrog/Verlet method was used to numerically integrate the equations of motion. Each run was at least 5000 ps with a time increment of $5 \mathrm{fs}$, the duration of which was determined such that the number of particles reaching the border plane was about 500 or more. The total number of particles in the cell, $\mathrm{N}$, ranged from 400 to 4200

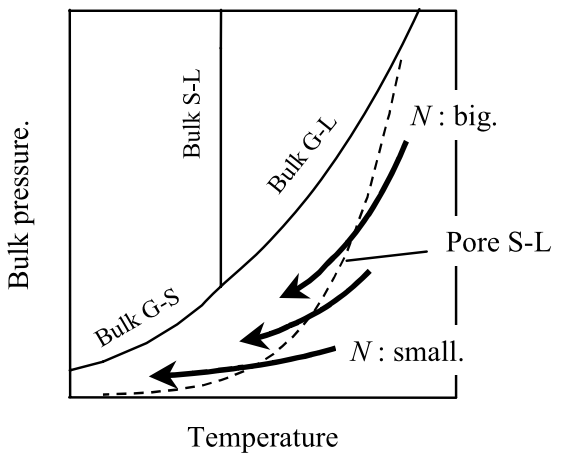

Fig. 2 Scheme of operating p-T conditions in the simulations

depending on the expected condition. The previous configuration, obtained at a higher temperature, was used for the initial configuration at a lower temperature. The operating $\mathrm{p}$ - $\mathrm{T}$ conditions in this sequence are the bold curves shown in Fig. 2. The equilibrium bulk pressure is higher for a larger value of $\mathrm{N}$.

\section{Results and discussion}

In this section, the variation of phase condition against temperature is explained, which exhibits liquid-solid phase transition at constant N. As explained in the authors' earlier study (Miyahara et al. 2000; Kanda et al. 2000, 2004, 2005), we are mainly interested in the freezing branch. The frozen particles have aligned in a concentric circles along the wall similar to those reported by Maddox and Gubbins (Maddox and Gubbins 1997). The freezing point was determined from the discrete changes in the density, enthalpy, arrangement, and structural functions against temperature (Kanda et al. 2000). Since the density $\rho^{*}$ of the adsorbate in the CFPF is a particularly easy to understand phase transition, the density profile during freezing is shown in Fig. 3. $\rho^{*}$ was determined as follows:

$\rho^{*}=\rho \sigma_{\mathrm{ff}}^{3} ; \quad \rho=\frac{\left\langle N_{C}\right\rangle}{D^{2} \pi / 4 \times 10 \sigma_{\mathrm{ff}}}$

where $\left\langle N_{C}\right\rangle$ is the ensemble average of the number of fluid particles in the CFPF. V is taken as the volume of the CFPF, which is determined as the space between the edges of the nuclei of the wall surface carbon atoms and includes some dead space in the vicinity of the wall where methane cannot penetrate due to the repulsive force. From this definition, the overall density is smaller than the true density of the pore fluid. As shown in Fig. 3, the density shows a gradual increase on cooling from a higher temperature. At the freezing point, a discrete change in density occurs, which is attributed to the latent heat, rearrangement of the molecules, in-plane pair correlation function, and structure factor (Kanda et al. 


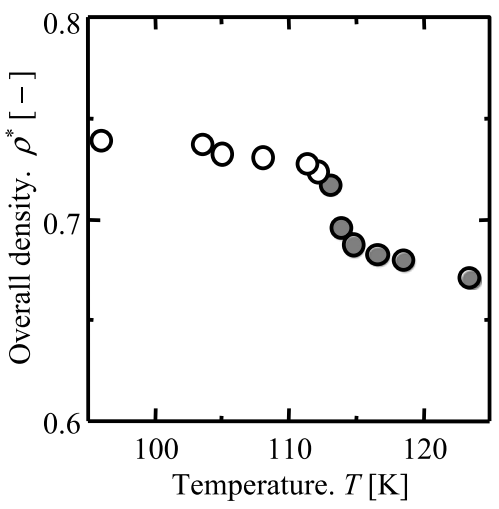

Fig. 3 Density profile within a pore of $D=7.5 \sigma_{\mathrm{ff}}$ at $N=1800$ with cooling. The open circles are solid phase and the closed ones are liquid phase

2000); thus, we decided the phase conditions based on the density change and the above factors. For $N=1800$ and $D=7.5 \sigma_{\mathrm{ff}}$, the density exhibits an almost vertical change at around $T=112-113 \mathrm{~K}$, while in the lower temperature range, a slight variation in the density is recognizable.

As shown in Fig. 4, the above-mentioned set of simulations was conducted for various values of $\mathrm{N}$ and the phase conditions were determined. Also, a similar series of simulations was conducted for the other sizes of the pores ranging from $5.5 \sigma_{\mathrm{ff}}$ to $9.5 \sigma_{\mathrm{ff}}$, as plotted in Fig. 5. The rather significant dependence of the freezing point on the bulkphase pressure below the saturated vapor pressure of the bulk phase is the most striking in this case. Normal bulk fluid shows only a slight change in the freezing point against the pressure as described by the almost vertical solid-liquid coexistence.

The authors earlier presented a simple model for the slitshaped nanopores; however, the model was verified only for the pores with the simplest geometries. Since the details of the model are given elsewhere (Miyahara et al. 2000), only the concept and outline of the model are briefly explained below. The surface of the pore phase exhibits a strong curvature when the pore phase is liquid-like, while it becomes rather flat with a solid-like phase under a high relative pressure. The strong Young-Laplace effect is assumed to exist in order to hold the melted phase with lower relative pressures. Thus, the observed depression in the freezing point below the saturated vapor line is considered to be accomplished with a significant change in the pressure on the capillary fluid. Based on the above concept, the basic equation to describe the freezing point depression is as follows:

$$
\begin{aligned}
\frac{k T}{\left(v_{L}\right)_{\text {bulk }}} \ln \frac{p}{p_{\mathrm{S}}(T)} & =\left.p^{\text {pore }}\right|_{T=T}-\left.p^{\text {pore }}\right|_{T=T_{a}} \\
& =\left(T-T_{a}\right)\left(\frac{\Delta s}{\Delta v}\right)_{\text {pore }}
\end{aligned}
$$

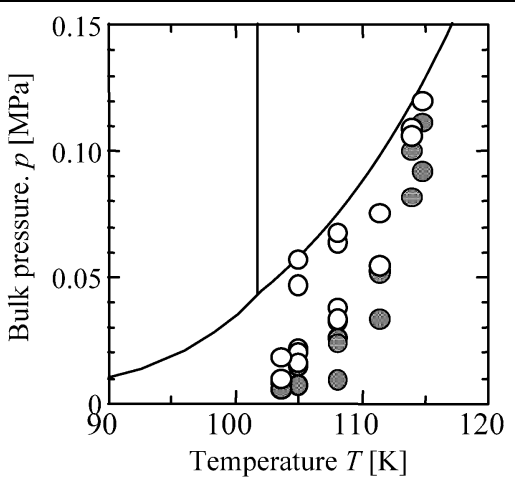

Fig. 4 Phase condition within a pore of $D=7.5 \sigma_{\mathrm{ff}}$ at several $N$. The open circles are solid phase and the closed ones are liquid phase

Table 1 Typical properties of solid-like (amorphous) phase in cylindrical nanopores

\begin{tabular}{llllll}
\hline$D$ & $v_{L}^{*}$ & $\Delta h^{*}$ & $\Delta v^{*}$ & $v_{L}^{*} \Delta h^{*} / \Delta v^{*}$ & $\alpha$ \\
\hline (Bulk) & 1.17 & 1.01 & 0.124 & 9.49 & 1.00 \\
$9.5 \sigma$ & 1.38 & 0.56 & 0.106 & 7.25 & 0.76 \\
$7.5 \sigma$ & 1.44 & 0.60 & 0.124 & 6.95 & 0.73 \\
$5.5 \sigma$ & 1.50 & 0.24 & 0.080 & 4.56 & 0.48 \\
\hline
\end{tabular}

$$
=\left(T-T_{a}\right) \alpha\left(\frac{\Delta s}{\Delta v}\right)_{\mathrm{bulk}},
$$

$p=p_{\mathrm{s}}(T) \exp \left(\frac{T-T_{a}}{T_{t}} \frac{\alpha}{k T}\left(\frac{v_{L} \Delta h}{\Delta v}\right)_{\text {bulk }}\right)$,

where $k$ is Boltzmann's constant; $p$, the vapor pressure; and the subscript $\mathrm{s}$, indicates saturation. $v_{L}$ is the volume per molecule of liquid, and $p_{\text {pore }}$ is the pressure of the pore phase. $T$ is the shifted freezing point relative to the freezing temperature $T_{a}$ at $p=p_{s} . \Delta s$ and $\Delta v$ are the entropy and molar volume, respectively, between the liquid and solid phases.

The above equation is easily understandable because it is the basis for the Kelvin equation. When the pressure difference is equated with the Young-Laplace equation, it yields the Kelvin equation. It should be noted here that (3) does not suffer from the incorrectness of the Kelvin equation for nanopores because it does not include any factor related to the pore size. Equation (3) can be converted to (4). $\Delta h$ and $T_{t}$ are the phase transition enthalpy and normal freezing point, respectively. Here, $\alpha$ indicates the ratio of the properties, $\left(v_{L} \Delta h\right) / \Delta v$, of the pore fluid against those of the bulk fluid. For the LJ bulk fluid, the properties such as $v_{L}, \Delta h$, and $\Delta v$ are well known as shown in Table 1 (Agrawal and Kofke 1995). Also, for the pore fluids, these properties are obtained in the previous study of the authors (Kanda et al. 2000). For example, the volume change and the latent heat are smaller for pores with smaller diameters. These data that derived $\alpha$ were smaller for smaller pores. 
Fig. 5 Liquid-solid coexistence curves of the pore fluid with various pore diameters obtained by MD simulations (keys) superimposed on the bulk phase diagram (thin solid lines). The bold solid lines are the predictions based on a simple model using the properties of the pore phase. The bold broken lines are the predictions based on a simple model using the properties of the bulk phase

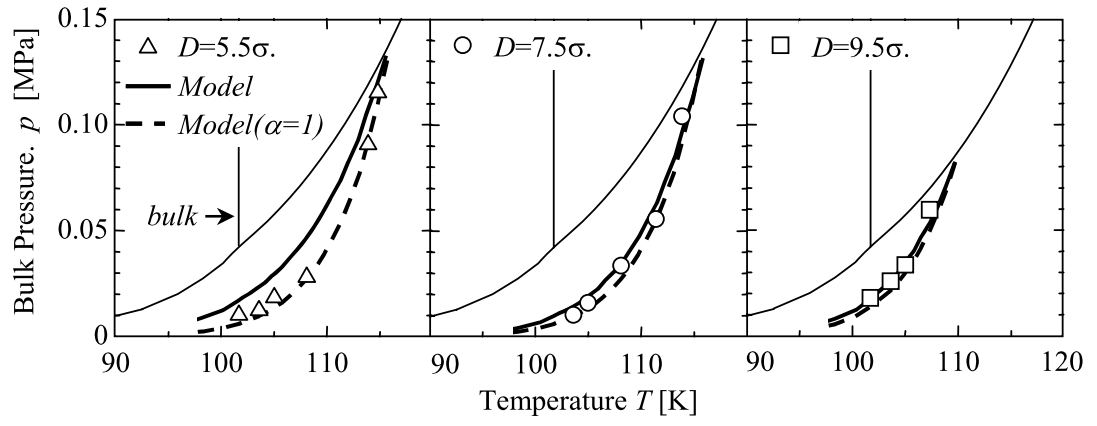

We examined the performance of this simple model by comparing it with the simulation results. $T_{a}$, the freezing point under saturated vapor, can be estimated based on the previous study of the authors (Kanda et al. 2000). The broken bold lines in Fig. 5 are the predictions of the assumption that $\alpha=1$, that is, the bulk properties. The solid bold lines are the predictions based on the properties of the pore fluid .

Surprisingly, at $D=7.5 \sigma$ and $9.5 \sigma$, the difference was insignificant. Also at $D=5.5 \sigma, \alpha$ does not cause a fatal error. Nevertheless, the simple model, presented for slitshaped pores using the bulk properties gives a reasonably good performance in expressing the freezing point shift for cylindrical nanopores as well.

\section{Conclusion}

The effect of the equilibrium vapor-phase pressure on freezing in a cylindrical pore was examined by employing MD simulations with an imaginary vapor phase. The MD simulations showed liquid-solid phase transitions with a variation in the equilibrium vapor-phase pressure below the saturated pressure. Thus, the determined solid-liquid coexistence line exhibited a significant dependence of the freezing point on a slight change in the bulk-phase vapor pressure; this implies the importance of the tensile effect on freezing in nanopores.

A simple model, which was earlier presented to describe the freezing point depression of slit-shaped nanopores, was derived in terms of the capillary effect that would reduce the effective pressure experienced by the pore fluid, and it showed a good agreement for cylindrical nanopores as well. Although in cylindrical pores, the structure of the solid phase exhibits a different nature as compared to that of bulk, the model is able to agree with the bulk properties of a simple fluid.

As a future approach, we would like to determine the triple point of the LJ fluid in cylindrical pores. We think that the pressure and temperature of the triple point must be significantly lower than that obtained in this study. For lowerpressure conditions, our MD simulation using the unique unit cell requires a long simulation time to count a sufficient number of escaped particles. Hence, we will attempt to further modify our MD technique to determine the triple point.

Acknowledgement This research was supported in part by the Grant-in-Aid for Scientific Research, No. 18033024, from the Ministry of Education, Culture, Sports, Science and Technology of Japan.

\section{References}

Agrawal, R., Kofke, D.A.: Mol. Phys. 85, 43-59 (1995)

Gelb, L.D., Gubbins, K.E., Radhakrishnan, R., Sliwinska-Bartkowiak, M.: Rep. Prog. Phys. 62, 1573-1659 (1999)

Kanda, H., Miyahara, M., Higashitani, K.: Langmuir 16, 8529-8535 (2000)

Kanda, H., Miyahara, M., Higashitani, K.: J. Chem. Phys. 120, 6173 $6179(2004)$

Kanda, H., Miyahara, M., Higashitani, K.: Adsorption 11, 295-299 (2005)

Kanda, H., Miyahara, M.: J. Chem. Phys. 126, 054703-1-0547034 (2007); (selected in Virtual Journal of Nanoscale Science \& Technology-February 12, 15(6) (2007))

Kaneko, K., Watanabe, A., Iiyama, T., Radhakrishnan, R., Gubbins, K.E.: J. Phys. Chem. B 103, 7061-7063 (1999)

Klein, J., Kumacheva, E.: Science 269, 816-819 (1995)

Klein, J., Kumacheva, E.: J. Chem. Phys. 108, 6996-7009 (1998)

Kofke, D.A.: J. Chem. Phys. 98, 4149-4162 (1993)

Kumacheva, E., Klein, J.: J. Chem. Phys. 108, 7010-7022 (1998)

Maddox, M.W., Gubbins, K.E.: J. Chem. Phys. 107, 9659-9667 (1997)

Miyahara, M., Gubbins, K.E.: J. Chem. Phys. 106, 2865-2880 (1997)

Miyahara, M., Kanda, H., Shibao, M., Higashitani, K.: J. Chem. Phys. 112, 9909-9916 (2000); (selected in Virtual Journal of Nanoscale Science \& Technology-June 8, 1(22) (2000))

Miyahara, M., Sakamoto, M., Kanda, H., Higashitani, K.: Stud. Surf. Sci. Catal. 144, 411-418 (2002)

Peterson, B.K., Walton, J.P.R.B., Gubbins, K.E.: J. Chem. Soc. Faraday Trans. 2 82, 1789 (1986)

Sliwinska-Bartkowiak, M., Dudziak, G., Sikorski, R., Gras, R., Gubbins, K.E., Radhakrishnan, R.: Phys. Chem. Chem. Phys. 3, 1179 (2001a)

Sliwinska-Bartkowiak, M., Radhakrishnan, R., Gubbins, K.E.: Mol. Simul. 27, 323 (2001b)

Watanabe, A., Iiyama, T., Kaneko, K.: Chem. Phys. Lett. 305, 71-74 (1999) 